\title{
Theory of the diffusion of heavy impurities in alkali metals
}

\author{
H Schober†, Roger Taylor +. M J Norgett and A M Stoneham \\ Theoretical Physics Division, AERE. Harwell, Didcot, Oxfordshire, OX11 ORA, UK
}

Received 15 November 1974

\begin{abstract}
We have developed interatomic potentials for heavy atom impurities in alkali metal hosts, and we have used them to calculate characteristic energies for the diffusion of gold in sodium and of silver in lithium. The calculations show that, whilst most impurity atoms should be present substitutionally, the diffusion is dominated by interstitial motion for both $\mathrm{Li}-\mathrm{Ag}$ and $\mathrm{Na}-\mathrm{Au}$. The large difference in observed behaviour stems from the different forms of the interatomic potentials, but cannot be described simply in terms of atomic radii or electronegativity arguments. The activation energies predicted are in good quantitative agreement with experiment.
\end{abstract}

\section{Introduction}

The properties of heavy impurities in alkali metals have been of interest recently for several different reasons. On the one hand, they are systems in which the relatively complex heavy atoms are embedded in a host with simpler electronic structure. The theory is tractable, and observations of properties sensitive to the electronic structure provide a check of treatments of impurity systems. On the other hand, the diffusion of heavy impurities is of interest, for the diffusion data can shed light on a number of aspects of the theory which are hard to analyse in other cases. These include questions of the relative merits of reaction rate theory and the dynamical theory (eg Mundy and McFall 1973) and the a priori calculation of heats of transport (eg Allnatt 1971). In addition, different systems behave in remarkably distinct ways: Ag in Li diffuses only slowly, whereas Au moves rapidly through the $\mathrm{Na}$ lattice. Various primitive arguments have been given to explain this, based on such aspects as atomic radii and electronegativity; we shall return to these criteria later.

In the present paper we consider two contrasted systems of heavy atoms in metals, namely $\mathrm{Li}-\mathrm{Ag}$ and $\mathrm{Na}-\mathrm{Au}$. After deriving suitable interatomic potentials for the interactions between the host atoms and between host and impurity we compare the energies for the various atomic configurations (eg interstitial or substitutional) and calculate activation energies for diffusion. We shall not discuss isotope effects in any detail, nor shall we discuss the heat of transport. However, it is hoped to return to these points in a later paper.

† Permanent address: Institüt für Festkörperforschung III. KFA. Jülich.. West Germany.

† Permanent address: Physics Division. National Research Council. Ottawa, Canada. 


\section{Interatomic potentials}

In calculating energies associated with isolated impurity atoms, we shall need the interaction potential $V_{\text {AA }}(r)$ between the host lattice atoms and the corresponding potential $V_{\mathrm{AB}}(r)$ between the impurity and the host atoms. For the two systems considered in this paper $(\mathrm{A}=\mathrm{Li}, \mathrm{B}=\mathrm{Ag}$ and $\mathrm{A}=\mathrm{Na}, \mathrm{B}=\mathrm{Au})$ the potentials have been calculated using conventional pseudopotential theory, in which the energy of the system is expanded to second order in the pseudopotential. Good agreement with the experimental phonon dispersion curves is obtained in both $\mathrm{Na}$ and $\mathrm{Li}$.

The host-host interactions, $V_{\mathrm{AA}}(r)$, have been calculated using the M2 model potential of Rasolt and Taylor (1974) for Na and the corresponding potential of Dagens et al (1974) for $\mathrm{Li}$; full details are reported in these two papers. The parameters of these model potentials have been adjusted so that the theory reproduces correctly the selfconsistent, nonlinear, charge density induced by an isolated ion placed in an electron gas of the same density as the corresponding metal. The use of this procedure incorporates all nonlinearities in the conduction electron response to single site scattering by the host lattice. The model potentials take the form:

$$
V_{\mathrm{M}}(r)=-\frac{Z e^{2}}{r}+\sum_{l=0}^{l=2}\left(A_{l}+\frac{Z e^{2}}{r}\right) \theta\left(R_{l}-r\right) \hat{P}_{l}
$$

where $\hat{P}_{l}$ is the angular momentum projection operator and $\theta(x)$ is the usual step function. The parameters $A_{l}$ and $R_{l}$ are listed in table 1 .

We do not have available selfconsistent nonlinear charge densities for isolated $\mathrm{Au}$ and $\mathrm{Ag}$ atoms in an electron gas of the appropriate alkali metal density. Hence we cannot include the nonlinearities in the electron response to these ions in our calculations. Instead, the parameters for the model potentials were determined by fitting to the spectroscopic terms of the free ions; spin-orbit interactions were ignored, the average level being used when the splitting was appreciable. This procedure is not valid for Ag metal or Au metal, of course, but is a reasonable approach for the problem in hand of an isolated impurity in an alkali metal. The parameters of model potentials for $\mathrm{Au}$ and $\mathrm{Ag}$ are also listed in table 1.

The interatomic potentials are obtained from the model potentials $V_{\mathbf{M}}(r)$ by methods described in detail by Rasolt and Taylor. The relevant expressions are given in their equations (25) and (43) for $V_{\mathrm{AA}}$, in (29) and (44) for $V_{\mathrm{AB}}$, and in (39) for the charge density. The Geldart and Taylor expressions for electron gas screening were used.

At this point it is instructive to examine the charge densities induced by isolated $A$ and $B$ ions placed in an electron gas of the metallic density. These are illustrated figure 1 . To a good approximation the metallic density may be constructed by linear

Table 1. Model potential parameters: the values listed here are the parameters of the model potential defined by (2.1). The $A_{l}$ are in Ryd and the $R_{l}$ in atomic units

\begin{tabular}{lcccccc}
\hline & $A_{0}$ & $A_{1}$ & $A_{2}$ & $R_{0}$ & $R_{1}$ & $R_{2}$ \\
\hline $\mathrm{Na}$ & -0.439 & -0.439 & -1.40 & 2.08 & 2.00 & 2.00 \\
$\mathrm{Li}$ & -0.70 & -1.32 & -1.32 & 2.38 & 2.38 & 2.38 \\
$\mathrm{Au}$ & -1.23 & -1.45 & -1.45 & 2.60 & 2.60 & 2.60 \\
$\mathrm{Ag}$ & -1.03 & -1.25 & -1.25 & 2.60 & 2.60 & 2.60 \\
\hline
\end{tabular}




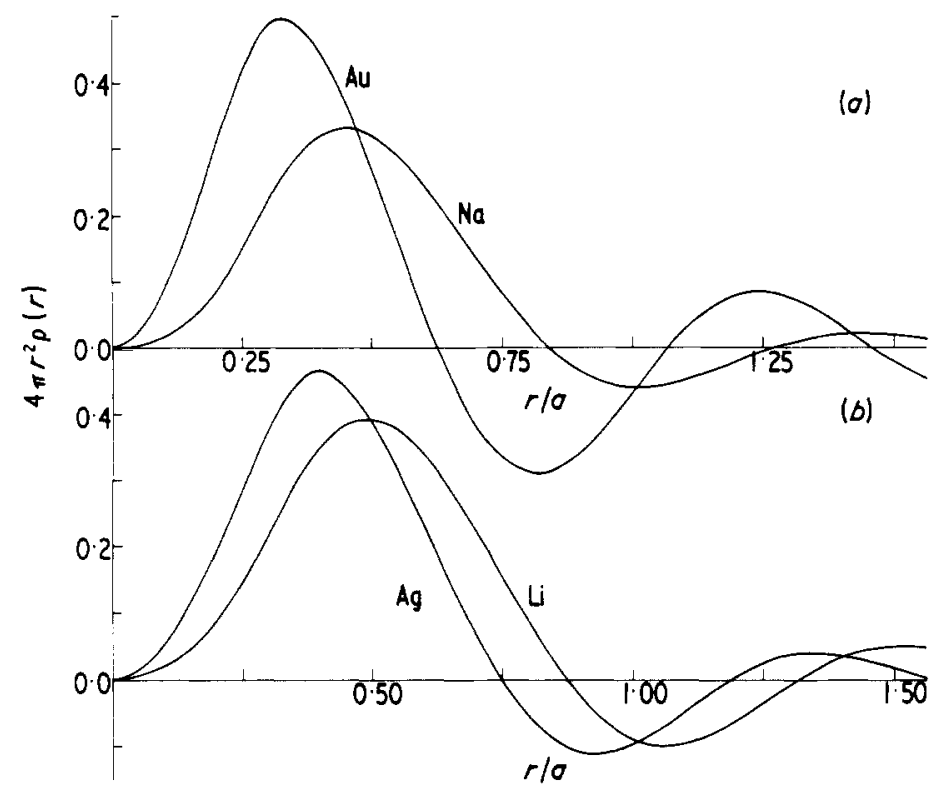

Figure 1. The charge densities $\rho(r)$ are shown as a function of $(r / a)$, the distance from the atom in units of the lattice parameter. $(a) 4 \pi r^{2} \rho(r)$ for $\mathrm{Na}$ and $\mathrm{Au}$ in $\mathrm{Na} ; a$ is $4.234 \AA$ and $r,=3.939$. (b) $4 \pi r^{2} \rho(r)$ for $\mathrm{Li}$ and $\mathrm{Ag}$ in $\mathrm{Li} ; a$ is $3.483 \AA$ and $r_{\mathrm{z}}=3.250$.

superposition of the isolated ion densitiest. It is interesting to note that in each case the charge is pulled in much closer to the impurity than the host ion. This has a profound effect on $V_{\mathrm{AB}}(r)$ as discussed later in this section. But before turning our attention to the potentials let us first comment on the atom size and electronegativity arguments. In the simplest atom size argument one would expect the impurity charge density to be scaled from that of the host, ie $\rho_{\mathrm{B}}(r) \simeq \rho_{\mathrm{A}}(\beta r)$ where $\beta$ is a scaling parameter. Judging by figure 1 this description is exceptionally bad for $\mathrm{Na}-\mathrm{Au}$ and although somewhat better for $\mathrm{Li}-\mathrm{Ag}$ it is still poor. Likewise the simplest electronegativity arguments would scale the charge density in a different way, ie $\rho_{\mathrm{B}}(r) \simeq \gamma \rho_{\mathrm{A}}(r)$. This description is completely unsatisfactory, for the maxima and minima of the host and impurity charge densities do not correspond. In short, one would not expect successful predictions from either the atom size or electronegativity arguments. Nor is it clear that a simple combination of them will be much more successful.

The interionic potentials are illustrated in figure 2 where it can be seen that in each case the first well of the impurity potential has a very deep minimum inside the first neighbour distance. This is clearly due to the increased charge at small $r$ commented upon earlier. As a result of this, the Coulomb field of the impurity ion is screened out more rapidly than is that of the host giving rise to more rapid onset of the long range oscillations in $V_{\mathrm{AB}}(r)$. The position of the deep minimum clearly plays an important role in determining the probability of interstitial formation.

Finally we note that the amplitudes of long range oscillations of the potentials decay like $r^{-n}$ for large $r$ where $n=5$ for the $\mathrm{Na}-\mathrm{Na}$ potential and $n=3$ for the others. For computation, we retained as much of the effect of these oscillations as was practical, and then brought the interaction potential smoothly to zero at a cutoff $R_{\mathrm{c}}$. In units of 

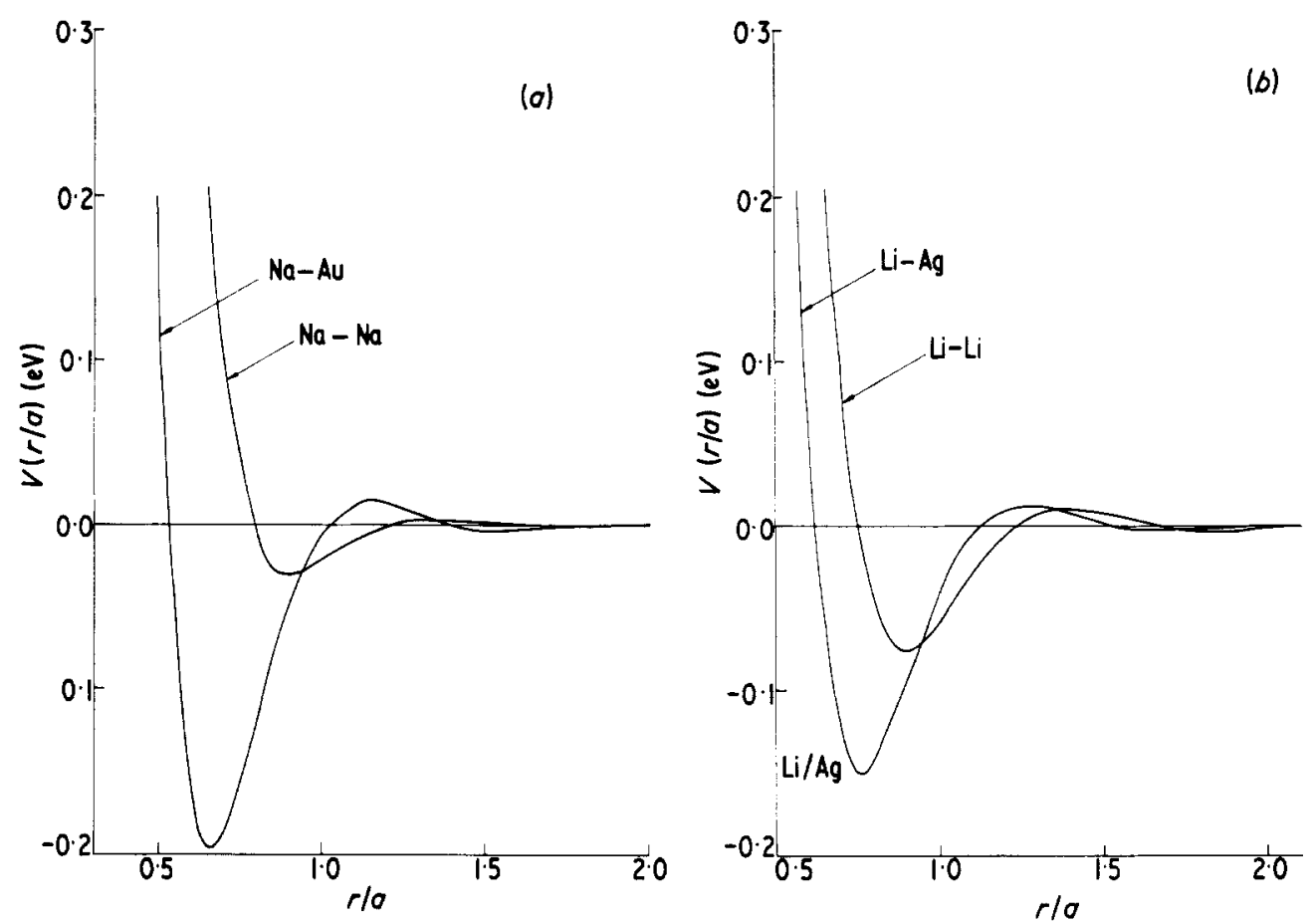

Figure 2. Interatomic potentials in electron volts as a function of $(r / a)$, a being the lattice parameter. (a) $V(r)$ for $\mathrm{Au}-\mathrm{Na}$ and $\mathrm{Na}-\mathrm{Na}$. (b) $V(r)$ for Ag-Li and $\mathrm{Li}-\mathrm{Li}$.

the lattice spacing (the cube edge of the host) the cutoffs chosen were $2 \cdot 12$ (Li-Li), 1.95 (Ag-Li), $1.97(\mathrm{Na}-\mathrm{Na})$ and $1.80(\mathrm{Au}-\mathrm{Na})$. We expect that corrections for the tails extending beyond these distances will be small.

\section{Calculation of lattice energies}

\subsection{Method}

The pair potentials obtained in $\$ 2$ were used to calculate the energies of defect configurations relevant to diffusion by vacancy and interstitial diffusion mechanisms. The calculations were done using the Harwell DEVIL program, a flexible package program designed for problems of this type. Vacancies, interstitials and substitutional impurities were inserted at appropriate places in the lattice. Lattice energies were calculated both before and after the lattice relaxation of a region containing the defects. This region consisted of a cubic block comprising eight $\{100\}$ planes in each direction; the relaxed region was surrounded by a rigid lattice region. A few calculations were performed using a much larger relaxed zone. Since the results were only changed by $0.004 \mathrm{eV}$, it is clear that the restriction on the relaxed region is not important.

The results are collected in table 2.

\subsection{Results for $\mathrm{Li}-\mathrm{Ag}$}

The predictions for both pure lithium and for $\mathrm{Li}-\mathrm{Ag}$ seem to be in good agreement with experiment. In pure lithium, the sum of the vacancy formation energy $(0.36 \mathrm{eV})$ and 
Table 2. Energies for various configurations of $\mathrm{Li}-\mathrm{Ag}$ and $\mathrm{Na}-\mathrm{Au}$. All energies are in $\mathrm{eV}$

\begin{tabular}{lcc}
\hline & $\mathrm{Li}-\mathrm{Ag}$ & $\mathrm{Na}-\mathrm{Au}$ \\
\hline Unrelaxed host vacancy $E_{\mathrm{VL}}$ & 0.82 & 0.32 \\
Relaxed host vacancy $E_{\mathrm{VR}}$ & 0.77 & 0.29 \\
Vacancy formation energy $\left(E_{\mathrm{VR}}-\frac{1}{2} E_{\mathrm{VL}}\right)$ & 0.36 & 0.13 \\
Vacancy saddle point energy & 0.88 & 0.38 \\
Interstitial impurity (relaxed) & -0.48 & -0.91 \\
$\quad \frac{1}{4}(111)$ midway between nearest neighbours & -0.41 & -0.96 \\
Tetrahedral & & -0.94 \\
$\quad$ Face centred & -0.43 & $-0.94 \dagger$ \\
Interstitial saddle point for lowest energy interstitial & -0.55 & $-0.89 \ddagger$ \\
Substitutional impurity & +0.56 & -0.27 \\
Saddlepoint for substitutional impurity & +0.30 & -0.58 \\
Substitutional impurity and neighbouring vacancy & & \\
\hline
\end{tabular}

† We checked a variety of interstitial positions, including some not listed. The likely saddle points were all around $-0.94 \mathrm{eV}$, and rather nearer $0.03 \mathrm{eV}$ than $0.02 \mathrm{eV}$ above the lowest state.

$¥$ The energies quoted in the text are the sums of these energies and $-\frac{1}{2} E_{\mathrm{vu}}$, the energy to put the host atom removed on the surface of the crystal. Thus, for $\mathrm{Li},-0.55-\frac{1}{2}(0.82)=$ $-0.96 \mathrm{eV}$, and for $\mathrm{Na},-0.89-\frac{1}{2}(0.32)=-1.05 \mathrm{eV}$.

activation energy for migration $(0.11 \mathrm{eV})$ is $0.47 \mathrm{eV}$, in good accord with the measured activation energy for self diffusion of $0.55 \mathrm{eV}$ (Lodding et al 1970).

The introduction of a silver atom at an interstitial site from infinity is an exothermic process, contributing $-0.48 \mathrm{eV}$, whilst the introduction of a substitutional silver atom contributes $-0.96 \mathrm{eV}$ after allowing for the displacement of a host atom to the surface. Thus Ag enters substitutionally into the lithium lattice, although the thermal population of the interstitial sites is important in diffusion.

The silver interstitial occupies a site at the centre of the join of two nearest neighbour host atoms. It can pass directly to an adjacent interstitial site over an energy barrier estimated at $0.05 \mathrm{eV}$. Since the fraction of silver atoms in interstitial solution is proportional to $\exp [+(0.48 \mathrm{eV}-0.96 \mathrm{eV}) / k T]$, an activation energy of $(0.96-0.48+$ $0.05) \mathrm{eV} \equiv 0.53 \mathrm{eV}$ is expected for interstitial diffusion. This is very close to the observed value of $0.56 \mathrm{eV}$ (Mundy and McFall 1973). The alternative vacancy mechanism gives a slightly higher activation energy. The impurity repels an adjacent vacancy with energy $0.08 \mathrm{eV}$, and requires $0.26 \mathrm{eV}$ for migration. Adding the vacancy formation energy $(0.36 \mathrm{eV})$ to these two gives a predicted activation energy of $0.70 \mathrm{eV}$. Whilst this is larger than the value $0.53 \mathrm{eV}$ for interstitial diffusion, the two energies are sufficiently close that both vacancy and interstitial mechanisms may operate at higher temperatures. This may lead to interesting temperature effects on the correlation factors. Mundy and McFall note, however, that there is no evidence of a temperature dependence of the observed activation energy.

\subsection{Results for $\mathrm{Na}-\mathrm{Au}$}

The predictions for pure sodium and for $\mathrm{Na}-\mathrm{Au}$ are only slightly less satisfactory. The central difficulty is that the $\mathrm{Na}$ model potential leads to only a low binding energy per atom $(0.32 \mathrm{eV})$ and hence to a very low vacancy formation energy $(0 \cdot 13 \mathrm{eV})$. Together with the migration energy of $0.06 \mathrm{eV}$, the predicted activation energy for self diffusion 
is only $0.19 \mathrm{eV}$, substantially less than the measured $0.45 \mathrm{eV}$ (Barr and Mundy 1965). We believe the main reason for the discrepancy comes from neglect of volume dependent terms. The formation energy should be enhanced by a term $\frac{1}{2}\left(C_{12}-C_{44}\right) V_{F}$, where $V_{\mathrm{F}}$ is the formation volume and $\frac{1}{2}\left(C_{12}-C_{44}\right)$ the Cauchy pressure. Whilst we have not calculated this term in detail, it seems probable that the correction will be larger for $\mathrm{Na}$ than for Li. This is suggested partly from the nature of the model potentials - for example there will be a term for $\mathrm{Li}$ from charge relaxation which will partly compensate the volume term-and partly from the experience of other workers. We are indebted to Dr M S Duesbery for the comment that the vacancy formation energy in $\mathrm{Na}$ is strongly volume dependent, a factor which makes direct comparison of our result with experiment difficult. Our prediction agrees well with the calculations of Ho (1973), apart from the question of volume dependent terms.

Despite the anomaly concerning pure sodium, the results for $\mathrm{Na}-\mathrm{Au}$ seem clear. The interstitial is much more stable than for $\mathrm{Li}-\mathrm{Ag}$, and there are a number of interstitial configurations with very similar energies. The most stable $\mathrm{Au}$ position seems to be the tetrahedral site, with energy $-0.96 \mathrm{eV}$. The activation energy could not be established with certainty, for there are several configurations close in energy. However, the interstitial migration energy cannot exceed $0.03 \mathrm{eV}$. The energy of a substitutional $\mathrm{Au}$ atom, $-1.05 \mathrm{eV}$, is only slightly less than that of the interstitial. Thus there will be a larger fraction of impurity interstitials in $\mathrm{Na}-\mathrm{Au}$ than in $\mathrm{Li}-\mathrm{Ag}$. The predicted activation energy for motion by an interstitial mechanism is $(1.05-0.96+0.03) \equiv 0.12 \mathrm{eV}$, which agrees well with the observed value of $0 \cdot 10 \mathrm{eV}$ (Barr et al 1969). A substitutional process would require $0.46 \mathrm{eV}$. Thus our calculations show clearly that different behaviour is expected for $\mathrm{Li}-\mathrm{Ag}$ and $\mathrm{Na}-\mathrm{Au}$, as well as giving good quantitative predictions.

\section{Discussion}

The predictions of $\$ 3$ successfully describe the observed activation energies for diffusion of the $\mathrm{Li}-\mathrm{Ag}$ and $\mathrm{Na}-\mathrm{Au}$ systems. The calculations indicate that the differences between these systems are quantitative rather than qualitative: in both cases the impurity diffusion occurs primarily by the motion of interstitials, even though many of the impurities enter substitutionally. We now examine other evidence to see if this description is satisfactory. Self diffusion in $\mathrm{Li}$ and $\mathrm{Na}$ is a much more controversial issue (Brown et al 1971), and we do not attempt to resolve it here.

Evidence for the interstitial or substitutional nature of the impurities is tentative. In $\mathrm{Na}-\mathrm{Au}$, where very fast impurity diffusion is seen, with a low activation energy, it is highly probable that interstitials dominate. That much gold is present interstitially is confirmed by centrifuge studies (Barr et al 1971). However, for $\mathrm{Li}-\mathrm{Ag}$ the activation energy is close to that for self diffusion. This is probably fortuitous, and it is interesting to note that earlier workers (Ott 1970, Titman and Moores 1972) have argued that the mechanism is at least partly interstitial. Titman and Moores' NMR study concluded that most of the silver a toms were present interstitially. However, their analysis assumed face centred interstitials, midway between (100) second neighbours, whereas our model predicts interstitials midway between (111) nearest neighbours. The local geometries differ, and rough estimates suggest that the Titman-Moores data can be fitted with a more modest fraction of interstitials. Thus the NMR data are consistent with our model, but not conclusive. It would be valuable to have channelling or centrifuge studies to establish more clearly the occupancy of the interstitial sites. 
We have not attempted a detailed calculation of the isotope effect. But it should be stressed that the diffusion mechanism we deduce is different from those considered by Mundy and McFall and that assumed by the theory they cite, so that the anomalies they find may not be significant. Further, the near degeneracy of the various interstitial positions in $\mathrm{Na}$-Au suggests that there should be a very low frequency resonance mode in this case. This resonance, and any related anharmonicity must be included in any calculations of isotope effects, for the interatomic forces are very different from those for a host atom in a perfect crystal. The resonance may also lead to a large sensitivity of the activation energy to hydrostatic pressure.

\section{Conclusions}

The success of our predictions suggests a number of conclusions. First, we believe we have verified our method of calculating the interatomic potentials for heavy impurities in an alkali metal host. Both the diffusion data and the experimental evidence concerning the site occupancies agree with the predictions based on the potentials. Secondly, whilst the potentials can vary appreciably from system to system, this variation cannot be described simply in terms of atomic radii or electronegativity arguments. Thirdly, the large differences in diffusion behaviour between $\mathrm{Li}-\mathrm{Ag}$ and $\mathrm{Na}-\mathrm{Au}$ do not come from major differences in mechanism, nor does the closeness of the activation energies of $\mathrm{Ag}$ and $\mathrm{Li}$ in $\mathrm{Li}$ imply a vacancy mechanism. For both $\mathrm{Li}-\mathrm{Ag}$ and $\mathrm{Na}-\mathrm{Au}$ diffusion is dominated by the fraction of the impurities which are interstitial, even though many of the impurities are substitutional. The differences in behaviour occur primarily because the interstitial sites are energetically less favourable for $\mathrm{Li}-\mathrm{Ag}$ than $\mathrm{Na}-\mathrm{Au}$, and this stems from the nature of the potentials.

We have not carried out detailed calculations of the intriguing isotope effects, but merely observe that previous calculations assume different diffusion mechanisms from those deduced here.

\section{Acknowledgments}

We have benefited from discussions with a number of our colleagues, including Mr A D Le Claire and Dr M Duesbery.

\section{References}

Allnatt A 1971 Z. Naturf. 26a 10-19

Barr L W. Le Claire A D and Smith F A 1971 Atomic Transport in Solids and Liquids ed A Lodding and T Lagerwall (Verlag) pp $336-41$

Barr L W and Mundy J N 1965 Diffusion on BCC Metals (Am. Soc. for Metals) p 171-83

Barr L W. Mundy J N and Smith F A 1969 Phil. Mag. 20389.98

Brown R C. Worster J. March N H. Perrin R C and Bullough R 1971 Phil. Mag. 23 555-76

Dagens L, Rasolt M and Taylor R 1974 Phys. Rev. to be published

Geldart D J W and Taylor R 1970 Can J. Phys. 48167

Ho P S 1973 Phys. Ret. B $73550-7$

Lodding A. Mundy J N and Ott A 1970 Phys. Stat. Solidi 38 559-69

Mundy J N and McFall W D 1973 Phis. Ret. B 7 4363-70 
Ott A 1970 Z. Naturf. 25a 1477

Perrin, Taylor and March 1975 to be published

Rasolt M and Taylor R 1974 Phys. Rer to be published

Titman J M and Moores B M 1972 J. Phy's. F: Metal Phys. 2 592-604 